\title{
Vitamin D Deficiency/Insufficiency among Inpatients with Depressive Symptoms
}

\author{
Young Sup Woo ${ }^{1}$, Sangha Kim ${ }^{1}$, Jong-Hyun Jeong ${ }^{1}$, Young-Eun Jung ${ }^{2}$, Moon-Doo Kim ${ }^{2}$, Won-Myong Bahk ${ }^{1}$ \\ ${ }^{1}$ Department of Psychiatry, College of Medicine, The Catholic University of Korea, Seoul, ${ }^{2}$ Department of Psychiatry, Jeju National University \\ School of Medicine, Jeju, Korea
}

\begin{abstract}
Objective: Although the association between low vitamin D levels and depressive symptoms has been widely reported, studies investigating the relationship between hypovitaminosis D and depressive symptomatology are scarce.

Methods: We retrospectively studied the relationship between vitamin D status and depressive symptoms in 196 patients hospitalized for a major depressive episode.

Results: The baseline 17-item Hamilton Depression Rating Scale total and depression factor (item 1, 2, 3, 7) scores were significantly higher in the vitamin-D-insufficiency/deficiency group than in the vitamin-D-sufficiency group.

Conclusion: It is important to consider measuring the vitamin D levels of patients with severe and core depressive symptoms and providing vitamin D supplementation when necessary.
\end{abstract}

KEY WORDS: Vitamin D; Depression; Anhedonia.

\section{INTRODUCTION}

Vitamin D deficiency/insufficiency is associated with both unipolar and bipolar depression. ${ }^{1,2)}$ Neurobiological and neuroendocrinological substrates have been identified that indicate a link between hypovitaminosis $\mathrm{D}$ and several psychiatric conditions, ${ }^{3,4)}$ including depression. ${ }^{5}$ Although many studies have investigated the link between vitamin $\mathrm{D}$ level and depression, research examining the relationship between hypovitaminosis $\mathrm{D}$ and the characteristics of depressive symptoms, such as subtype or symptom cluster, in patients with depression is scarce. Because not all depressed patients may be deficient or insufficient in vitamin $\mathrm{D}$, the characterization of the type of depression associated with vitamin $\mathrm{D}$ insufficiency/deficiency is of clinical importance. Therefore, we aimed to investigate the relationship between vitamin $\mathrm{D}$ status and

Received: January 3, 2018 / Revised: January 11, 2018

Accepted: January 14, 2018

Address for correspondence: Won-Myong Bahk, MD, PhD

Department of Psychiatry, Yeouido St. Mary's Hospital, College of

Medicine, The Catholic University of Korea, 10 63-ro,

Yeoungdeungpo-gu, Seoul 07345, Korea

Tel: +82-2-3779-1250, Fax: +82-2-780-6577

E-mail: wmbahk@catholic.ac.kr

ORCID: https://orcid.org/0000-0002-0156-2510 the specific characteristics of depressive symptoms according to Diagnostic and Statistical Manual of Mental Disorders, 5th edition (DSM-5) specifiers for mood episodes as well as the 17-item Hamilton Depression Rating Scale (HDRS) definitions of symptom clusters.

\section{METHODS}

\section{Patients and Assessments}

This study was retrospective; we reviewed the medical records of patients who were hospitalized in the psychiatric wards of three university hospitals in Korea. All patients had a diagnosis of major depressive disorder, bipolar I disorder, or bipolar II disorder. The patients were 19 years of age or older and were diagnosed clinically as having a major depressive episode (MDE) according to DSM- 5 criteria at the index hospitalization. Patients with insufficient data and those who had a severe comorbid medical or neurological condition that could contribute to depressive symptoms were excluded from the analysis. Patients who were known to have received vitamin D supplements were excluded. The charts of 245 subjects were reviewed, and 49 cases were excluded based on the aforementioned criteria. In total, 196 inpatients with a

(c) This is an Open-Access article distributed under the terms of the Creative Commons Attribution Non-Commercial License (http://creativecommons.org/licenses/by-nc/4.0) which permits unrestricted non-commercial use, distribution, and reproduction in any medium, provided the original work is properly cited. 
MDE were enrolled in the study and categorized into two groups according to the blood levels of $25(\mathrm{OH}) \mathrm{D}$ : vitamin D sufficiency $(\geq 30 \mathrm{ng} / \mathrm{ml})$ and insufficiency/deficiency $\left.(<30 \mathrm{ng} / \mathrm{ml}){ }^{6}\right)$

The patients' medical records were reviewed to examine their sociodemographic and clinical characteristics, including age, sex, socioeconomic status (SES), number of MDEs, duration of illness, severity of MDEs, and body mass index. DSM-5 specifiers at the index hospitalization, including mixed features, psychotic features, anxious distress, atypical features, melancholic features, and seasonal pattern, were also examined. The HDRS and 25(OH)D blood levels obtained within the first 3 days of the index hospitalization were collected from hospital charts and electronic medical records. HDRS items were grouped into four separate factors based on the clustering described by Shafer ${ }^{7)}$ : anxiety (items 9, 10, 11, 15, and 17), depres- sion (items 1, 2, 3, 7, and 8), insomnia (items 4, 5, and 6), and somatization (items 12,13,14, and 16). As this was a retrospective study, all data were obtained during routine psychiatric examinations and treatment.

\section{Statistical Analysis}

Statistical analyses were preformed using SAS for Windows (version 9.2; SAS Institute, Cary, NC, USA). For the univariate comparisons between the sufficiency group and the insufficiency/deficiency group, we used chisquare or Fisher's exact tests for categorical variables, and independent $t$ tests were used for continuous variables. Logistic regression was used for the adjusted comparison between the insufficiency/deficiency and sufficiency groups with regard to categorical variables. An analysis of covariance (ANCOVA) was performed to compare the HDRS total and cluster scores after adjusting for potential

Table 1. Demographic and clinical characteristics in relation to vitamin D status

\begin{tabular}{|c|c|c|c|}
\hline Characteristic & Insufficiency/deficiency $(n=164)$ & Sufficiency $(n=32)$ & $p$ value \\
\hline Male & $45(27.4)$ & $13(40.6)$ & 0.135 \\
\hline Age (yr) & $53.5 \pm 18.7$ & $49.0 \pm 18.5$ & 0.214 \\
\hline \multicolumn{4}{|l|}{ SES } \\
\hline Low & $9(5.5)$ & $0(0)$ & 0.371 \\
\hline Middle & $149(90.9)$ & $27(84.4)$ & 0.268 \\
\hline High & $6(3.7)$ & $5(15.6)$ & $0.007^{*}$ \\
\hline Number of depressive episodes & $2.8 \pm 2.6$ & $3.4 \pm 2.4$ & 0.255 \\
\hline Unipolar depression & $143(87.2)$ & $25(78.1)$ & 0.180 \\
\hline Duration of illness (yr) & $7.3 \pm 8.5$ & $8.3 \pm 7.5$ & 0.547 \\
\hline Severity of depressive episode & & & 0.337 \\
\hline Mild & $4(2.4)$ & $0(0)$ & \\
\hline Moderate & $33(20.1)$ & $10(31.3)$ & \\
\hline Severe & $127(77.4)$ & $22(68.8)$ & \\
\hline \multicolumn{4}{|l|}{ Subtype of current depressive episode } \\
\hline With mixed features & $12(7.3)$ & $2(6.3)$ & 0.830 \\
\hline With psychotic features & $83(50.6)$ & $24(75.0)$ & $0.011 *$ \\
\hline With anxious distress & $18(11.0)$ & $3(9.4)$ & 0.789 \\
\hline With atypical features & $21(12.8)$ & $6(18.8)$ & 0.372 \\
\hline With melancholic features & $19(11.6)$ & $2(6.3)$ & 0.372 \\
\hline With seasonal pattern & $4(2.4)$ & $1(3.1)$ & 1.000 \\
\hline BMI $\left(\mathrm{kg} / \mathrm{m}^{2}\right)$ & $23.7 \pm 3.7$ & $24.2 \pm 3.7$ & 0.479 \\
\hline Obesity (BMI $\geq 25$ kg/m²) & $105(64.0)$ & $19(59.4)$ & 0.618 \\
\hline Winter season (October-March) & $62(37.8)$ & $8(25.0)$ & 0.167 \\
\hline \multicolumn{4}{|l|}{ HDRS score } \\
\hline Total score & $24.0 \pm 6.3$ & $21.6 \pm 4.9$ & $0.037^{*}$ \\
\hline Anxiety factor (item 9, 10, 11, 15, 17) & $9.2 \pm 2.9$ & $9.2 \pm 2.9$ & 0.918 \\
\hline Depression factor (item 1, 2, 3, 7, 8) & $7.7 \pm 2.7$ & $6.5 \pm 2.3$ & $0.022^{*}$ \\
\hline Insomnia factor (item 4, 5, 6) & $3.5 \pm 1.7$ & $2.8 \pm 1.5$ & 0.061 \\
\hline Somatization factor (item 12, 13, 14, 16) & $3.4 \pm 1.7$ & $2.8 \pm 1.5$ & 0.074 \\
\hline
\end{tabular}

Values are presented as number (\%) or mean \pm standard deviation.

SES, socioeconomic status; BMI, body mass index; HDRS, 17-item Hamilton Depression Rating Scale. $* p<0.05$. 
confounders.

\section{Ethics}

This study was conducted in accordance with the Declaration of Helsinki, and approval to conduct the chart review was obtained from the Institutional Review Board. Because this was a retrospective study and data were obtained during routine psychiatric examination and treatment, the Board determined that informed consent was unnecessary.

\section{RESULTS}

Of the 196 patients included in the study, 164 (83.7\%) showed vitamin $D$ insufficiency/deficiency. The mean $25(\mathrm{OH}) \mathrm{D}$ blood level in the insufficiency/deficiency group was $17.1 \pm 7.1 \mathrm{ng} / \mathrm{ml}$, and it was $40.7 \pm 10.3 \mathrm{ng} / \mathrm{ml}$ in the sufficiency group. The subject distributions in the sufficiency and insufficiency/deficiency groups are shown in Table 1. Patients in the sufficiency group were more likely to have high SES $(p=0.007)$ and psychotic features $(p=0.011)$ compared with the insufficiency/deficiency group. The odds ratio for vitamin D sufficiency according to psychotic features was 3.43 (95\% confidence interval=1.39-8.46; $p=0.008$ ) when adjusted for covariates including age, sex, SES and baseline HDRS total score. The proportion of patients who met the DSM-5 criteria for mixed features, anxious distress, atypical features, melancholic features, or seasonal pattern did not significantly differ between the two groups.

The baseline HDRS total score was significantly higher $(24.0 \pm 6.3)$ in the insufficiency/deficiency group compared with the sufficiency group $(21.6 \pm 4.9 ; p=0.037)$, and this significance remained after adjusting for confounding variables, including age, sex, SES, and psychotic features ( $\mathrm{F}=4.650 ; p=0.032)$. Of the four HDRS factor scores, the depression factor score was significantly higher in the insufficiency/deficiency group $(7.7 \pm 2.7)$ compared with the sufficiency group $(6.5 \pm 2.3 ; p=0.022)$. The factor scores for insomnia (3.5 \pm 1.7 vs. $2.8 \pm 1.5 ; p=0.061$ ) and somatization $(3.4 \pm 1.7$ vs. $2.8 \pm 1.5 ; p=0.074)$ were numerically higher in the insufficiency/deficiency group compared with the sufficiency group; however, the difference was not significant. After using an ANCOVA to control for age, sex, SES, presence of psychotic features, and baseline HDRS score, the only factor that continued to yield a statistically significant difference between groups was depression ( $\mathrm{F}=4.272 ; p=0.040)$.

\section{DISCUSSION}

In the current study, only $16.3 \%$ of patients had sufficient vitamin D levels. This result is similar to a previous report in which $12.6 \%$ of hospitalized patients with depression had sufficient vitamin D levels ${ }^{8)}$ and suggests that low vitamin D levels are associated with severe depression that requires hospitalization. Moreover, the vitamin $D$ insufficiency/deficiency group showed more severe depressive symptoms on the HDRS. This is also consistent with previous studies that reported an inverse association between vitamin D level and depressive symptom severity as determined by self-rated instruments. ${ }^{8,9)}$

We found that symptoms related to the HDRS factor reflecting core depressive symptoms were significantly increased in patients with insufficient/deficient vitamin D compared with those having sufficient vitamin D; however, the subtype of depression, as determined by DSM-5 specifiers (except "with psychotic features"), was not associated with vitamin D status. In a previous study, low vitamin D levels were associated with cognitive/affective symptoms and anhedonia. ${ }^{8)}$ Because anhedonic features and cognitive symptoms, such as decision-making difficulties, are nested within the work/activities item (item 7) in the depression factor of the HDRS, ${ }^{10)}$ the results from the present study are consistent with the those of the previous study and suggest a relationship between hypovitaminosis $\mathrm{D}$ and the core symptoms of depression. We unexpectedly found that psychotic symptoms were more prevalent in patients with sufficient vitamin D compared with patients in the insufficiency/deficiency group. These data may indicate that depressive symptoms tend not to be severe enough to require hospitalization in the absence of psychotic symptoms in patients with a sufficient vitamin D level. It is also noteworthy that neither the subtype of depression, determined using DSM-5 specifiers, nor the polarity of depression (bipolar or unipolar) was affected by vitamin D status.

The main limitation of our study is the small sample size. We could not separate groups based on vitamin D insufficiency and deficiency due to sample size limitations; therefore, the statistical power may not have been great enough to detect smaller differences between 
groups. Furthermore, we did not evaluate behavioral aspects, such as physical activity, sun exposure, or dietary sources of vitamin $D$, which could affect circulating vitamin D levels. Finally, the effects of medications and psychiatric comorbidities were not determined. Despite these limitations, the results of the present study provide useful information indicating the association of vitamin $D$ insufficiency/deficiency with severity and type of depressive symptoms in patients with MDE. This might suggest that clinicians should consider the possibility of usefulness of vitamin D supplementation for patients with severe and core depressive symptoms.

\section{REFERENCES}

1. Anglin RE, Samaan Z, Walter SD, McDonald SD. Vitamin D deficiency and depression in adults: systematic review and meta-analysis. Br J Psychiatry 2013;202:100-107.

2. Berk M, Sanders KM, Pasco JA, Jacka FN, Williams LJ, Hayles $\mathrm{AL}$, et al. Vitamin D deficiency may play a role in depression. Med Hypotheses 2007;69:1316-1319.

3. Adamson J, Lally J, Gaughran F, Krivoy A, Allen L, Stubbs B. Correlates of vitamin $D$ in psychotic disorders: A comprehensive systematic review. Psychiatry Res 2017;249:78-85.
4. Altun $\mathrm{H}$, Kurutaş EB, Şahin N, Güngör O, Fındıklı E. The levels of vitamin D, vitamin D receptor, homocysteine and complex $B$ vitamin in children with autism spectrum disorders. Clin Psychopharmacol Neurosci 2018;16:383-390.

5. Eyles DW, Burne TH, McGrath JJ. Vitamin D, effects on brain development, adult brain function and the links between low levels of vitamin $D$ and neuropsychiatric disease. Front Neuroendocrinol 2013;34:47-64.

6. Holick MF. Vitamin D deficiency. N Eng/ J Med 2007;357: 266-281.

7. Shafer AB. Meta-analysis of the factor structures of four depression questionnaires: Beck, CES-D, Hamilton, and Zung. I Clin Psychol 2006;62:123-146.

8. von Känel R, Fardad N, Steurer N, Horak N, Hindermann E, Fischer $\mathrm{F}$, et al. Vitamin D deficiency and depressive symptomatology in psychiatric patients hospitalized with a current depressive episode: a factor analytic study. PLoS One 2015; 10:e0138550.

9. Milaneschi Y, Hoogendijk W, Lips P, Heijboer AC, Schoevers $\mathrm{R}$, van Hemert AM, et al. The association between low vitamin $D$ and depressive disorders. Mol Psychiatry 2014;19:444451.

10. Bagby RM, Ryder AG, Schuller DR, Marshall MB. The Hamilton Depression Rating Scale: has the gold standard become a lead weight? Am J Psychiatry 2004;161:2163-2177. 